\title{
Prevalence of Under Nutrition and its Associated Factors Among Pregnant Women in Konso District, Southern Ethiopia: Community Based Cross Sectional Study
}

\section{DEYGANTO GELEBO}

Save the children international

MATHEWOS GEBREMICHAEL ( $\sim$ alemumathewos2017@gmail.com )

Arba Minch University

GISTANE ASALE

Arba Minch University

DESSALEGN BERBADA

Arba Minch University

\section{Research Article}

Keywords: Under nutrition, Maternal malnutrition, Pregnant women, Konso, Ethiopia

Posted Date: January 19th, 2021

DOl: https://doi.org/10.21203/rs.3.rs-145846/v1

License: (c) (1) This work is licensed under a Creative Commons Attribution 4.0 International License.

Read Full License

Version of Record: A version of this preprint was published at BMC Nutrition on July 12th, 2021. See the published version at https://doi.org/10.1186/s40795-021-00437-z. 


\title{
Prevalence of Under Nutrition and its Associated Factors among Pregnant Women in Konso District, Southern Ethiopia: Community based cross sectional study
}

\author{
DEYGANTO GERGITO GELEBO ${ }^{1}$, MATHEWOS ALEMU GEBREMICHAEL ${ }^{2 *}$, \\ GISTANE AYELE ASALE ${ }^{2}$, DESSALEGN AJEMA BERBADA ${ }^{2}$ \\ ${ }^{1}$ Save the Children International, Konso Project Office, Ethiopia. \\ ${ }^{2}$ School of Public Health, College of Medicine and Health Sciences, Arba Minch \\ University, Arba Minch, Ethiopia.
}

*Corresponding author: Mathewos Alemu Gebremichael, School of Public Health, College of Medicine and Health Sciences, Arba Minch University, Arba Minch, Ethiopia, Tel: +251917784364, E-mail: alemumathewos2017@gmail.com

\begin{abstract}
Background: Under nutrition during pregnancy is an important public health problem. It is highly prevalent in Ethiopia but not sufficiently addressed yet. Hence, this study aimed to assess the prevalence of under nutrition and its associated factors among pregnant mothers in Konso district, Ethiopia.

Methods: Community based cross-sectional study was conducted with qualitative inquiry study from December 2018 to January 2019. A multistage stratified sampling technique was used to select 527 subjects. Quantitative data were collected using pretested structured questionnaire. The qualitative data were generated through two focus group discussions among purposely selected discussants. Mid upper arm circumference (MUAC) was measured by standard non stretchable MUAC tape. Data were entered into Epi-data version3.1 and analyzed by SPSS version 21. In multivariable binary logistic regression statistically significant association was declared at $\mathrm{p}$-value $<0.05$ while thematic framework analysis was employed for the qualitative data.
\end{abstract}

Results: Among 501 participants, the overall prevalence of under nutrition was $43.11 \%$ (95\% CI 38.76\%-47.47\%). Family food insecurity (AOR = 3.1; 95\%CI: 2.1-4.6), low dietary diversity score $(\mathrm{AOR}=4.9 ; 95 \% \mathrm{CI}$ : 2.6-9.2), medium dietary diversity score $(\mathrm{AOR}=2.3$; 95\%CI: 1.2-4.7), absence of latrine (AOR $=1.8 ; 95 \% \mathrm{CI}: 1.2-2.6)$ and having family resource decision making by husband only (AOR $=1.7$; 95\%CI: 1.1-2.6) were significantly associated factors. Traditional believes to restrict food such as egg, milk and milk products, avocado for women, weak nutrition education and malnutrition screening program, daily consumption of locally prepared alcoholic drink called "Cheka", draught prone nature of this setting, 
traditional way of farming practices and low socio-economic status were found to be barriers for women's under nutrition.

Conclusions: The prevalence of under nutrition was found to be higher than previously reported findings. Food security, dietary diversity, latrine availability, family resource decision making, food restrictions, weak maternal nutrition education and malnutrition screening program, practice of depending on local alcoholic drink called "Cheka", draught, traditional way of farming and low socio-economic status were identified factors. Hence, interventions targeting maternal nutrition education, hygiene and sanitation promotion, house hold food insecurity improvement strategies should be implemented to improve nutritional status of pregnant women.

Key words: Under nutrition, Maternal malnutrition, Pregnant women, Konso, Ethiopia

\section{Background}

Under nutrition is an important public health issues particularly for vulnerable groups including children and women of child bearing age especially pregnant mothers (1). Under Nutrition is a serious global health problem. About 795 million people are under nourished mostly in low and middle income countries and the problem is most critical during pregnancy (2). Globally, under nutrition is contributing for the deaths of 3.5 million of mothers and under five years of age children each year. It is estimated that 13 million children are annually born with IUGR (intra uterine growth retardation), 112 million are underweight secondary to under nutrition during pregnancy $(3,4)$.

Pregnancy is a critical phase in which mothers need optimal nutrients of good qualities of food to support the developing fetus (5).Adequate supply of nutrients and oxygen for the mother to her fetus is one of the factors that is critical for fetal survival . The ability of the mother to provide nutrients for her baby depends up on the nutritional status ,body size and body composition of the mother and all of which are being established throughout the life of her fetus (6).

Under nutrition in pregnant mother is a key contributor to many problems. It makes the women more susceptible to diseases, more risk of having miscarriages, poor fetal growth, low birth weight, infant morbidity and mortality. It can also cause long term irreversible and detrimental cognitive motor and health impairment (7). In addition to serious consequences of a health, the economy can be also affected by undernutrition. High prevalence of undernutrition can hampers economic growth and preserves poverty both directly, through a loss of productivity due to poor physical condition, and indirectly, through poor cognitive function and learning deficits $(8,9)$. 
Evidences showed that the burden of under nutrition among pregnant women is high. The prevalence of pregnant women's undernutrition in India 5\%, China 21\%, Sirlanka 15\%, Nigeria 10-40\% (10-12). In Ethiopia, the prevalence of under nutrition in pregnant women varies from 9.2\%-34\%. It was reported in Central Refit Valley of Ethiopia, Wondogenet District in Ethiopia, Silte zone, Southern Ethiopia, Dessie Northern Ethiopia, Eastern Ethiopia, Gondar town and hospital in Northern Ethiopia, humanitarian setting of Ethiopia, Shashemene, $31.7 \%, 9.2 \%, 21.8 \%, 19.8 \%, 19.5,16.2 \%, 14.4 \%, 24 \%, 34 \%$ and $35.5(13-21)$. The most acceptable explanation for this wide variation is likely to be the fact that variation in the contextual factors of pregnant women's undernutrition.

Previous studies identified different risk factors for pregnant women under nutrition. These factors were: age, educational status, family size,, marital status, social class, height of the mother, house hold food insecurity, house hold asset, homeownership, house hold saving, financial constraint, ownership of life stock, job loss and low level of income, gender of house hold head, cultivated farm land size, inadequate dietary intake, women's knowledge about nutrition, residence, iron supplementation during pregnancy, meal frequency per day, trimester, perceptions imposing dietary restriction on certain food, health status of pregnant mothers, antenatal care service dissatisfaction, and family planning utilization before current pregnancy $(11,13,22-31)$.

Due to differences in characteristics of socio-economic, culture, ethnicity, and geographical location, associated factors for undernutrition in pregnant women might not be the same across different regions of Ethiopia. The government of Ethiopian launches a national nutrition program. Maternal nutrition is one of its targets needs updated data which is essential to develop effective intervention strategies to prevent undernutrition of pregnant women and children. In addition to this, there is limited data on prevalence and factors associated with maternal undernutrition particularly in the southern part of Ethiopia. According to 2010 E.C Konso district health office report, the prevalence of under nutrition among pregnant mothers was more than $30 \%$ (32), but the risk factors were not well understood. And also the cultural practices which restrict some important foods during pregnancy are commonly practiced in Konso. Therefore, undernutrition of pregnant women need to be assessed in a specific context to develop effective interventions. Hence, this study determined the prevalence undernutrition and its associated factors among pregnant women in Konso district, Southern Ethiopia. 


\section{Methods}

\section{Study Area, Study Design and Study Period}

The community based cross sectional study design with focus group discussion (FGD) was conducted in Konso District from $1^{\text {st }}$ December, 2018 to $31^{\text {st }}$ January, 2019. Konso District is located at 595 Kilometers from Addis Ababa (capital city of Ethiopia) and $365 \mathrm{~km}$ from Hawassa (the capital city of south region). Based on the Konso district administrative population profile the district has a total population of 270,837 from this 132,710 male and female 138,127. The District has 50 health posts, 11 health centers, and 1 primary hospital and 25 private clinics which are providing the health service for the community.

\section{Population}

The source population of this study was all pregnant women in Konso District. The study population was all pregnant women in the selected kebeles (small administrative unit in Ethiopia) of Konso District. All pregnant women who were residents of Konso district for at least six months or above were included to the study whereas those pregnant women who were seriously ill, those who had hand deformity were excluded.

\section{Sample size determination and sampling technique}

The sample size was computed using single population proportion formula; considering $95 \%$ confidence level, 5\% margin of error, 1.5 design effect, $31.8 \%$ hypothesized prevalence of under nutrition among pregnant women which has been taken from a study conducted in central rift valley of Ethiopia (13) and by adding 5\% none response rate the final sample size for this study was 527. A multi-stage stratified sampling which was followed by a systematic sampling technique was applied to reach each study participants. The kebeles in the district were stratified in to rural and urban. One urban and eight rural kebeles were selected by using lottery method from a total of forty one kebeles in Konso district. Then by using Health Extension Workers Housing Registration, the total number of households with pregnant women $(2,277)$ was accessed and the sampling interval was calculated. Finally households with eligible pregnant women were selected using a systematic random sampling technique. For those house holds with more than one pregnant women, one pregnant woman was selected by using the lottery method. Data collators visited the house on the next day, when the pregnant women were not available at home and the pregnant women who were not available during second visit were recorded as non-response and then nearby household was considered. 


\section{Qualitative sampling procedure}

Two FGD was conducted with 12 subjects in each group who were purposively selected. Situational analysis was done before conducting the focus group discussion (FGD) to minimize errors on selection of participants. The key informants were a case team leader for maternal health and a supervisor assigned for each kebele and agricultural expert (agriculture and natural resource head). In each FGD pregnant women, their husbands, and health extension workers participated. To minimize the possible bias on selection of the study participants, we made sure to emphasize that we want a group of people that can express a range of views, to be able to have a proper discussion. Smooth discussion environment was created and we tried to encourage the communication and interaction during the focus group discussion in every possible way. FGDs were held in a neutral setting which encourages participants to express their views freely. We made sure that there were no disturbances, adequate lighting and ventilation as it was the hottest season during data collection period and also there were cold beverages including water. Materials which were necessary to conduct the focus group discussion (FGD) were prepared before discussion like FGD guide line, voice recorders, note books, pen and pencils. To create conducive discussion area, the chairs were arranged in a circle

\section{Study variables}

The dependent variable in this study was under nutrition among pregnant women. The independent variables were: Socio-demographic factors:- age, marital status, husband education maternal education, family size, polygamy. Maternal related factor: - parity, family planning utilization before current pregnancy, birth interval, receiving iron supplementation, ANC follow up, ANC satisfaction, Nutrition knowledge, Illness, History of abortion, History of still birth, dietary diversification (24-hour recall), meal frequency, Socio Cultural factors: food taboo and food restriction during pregnancy, decision making on household assets, family stable food. Economic factors:-Family source of food, Farm land owner ship, Employment (Maternal\& husband job) status, House hold income, Wealth index. Hygiene and Sanitation related factors:-access to water and sanitation facilities, such as latrine availability \&utilization, Family source of water, distance to get water. Food Security related factors - food accessibility and availability. 


\section{Operational Definitions}

Under Nutrition: - Pregnant mother whose Mid-upper arm circumference (MUAC) less than $23 \mathrm{~cm}$ is consider as under nutrition (33).

Food-secure: A household was labeled 'food secure' when the members 'rarely', in the past four weeks, worried about not having enough food and had replied 'no' to question number 2 to 9 of HFIAS $(34,35)$.

Food restriction:-Avoidance of any kind of food during pregnancy

Mildly food-insecure: The members of the household worried about not having enough food sometimes or often, and/or were unable to eat preferred foods, and/or ate a more monotonous diet than desired, and/or ate some foods considered undesirable but only rarely(35).

Moderately food-insecure: The household members sacrificed quality more frequently by eating a monotonous diet or undesirable foods sometimes or often, and/or had started to cut back on quantity by reducing the size of meals or number of meals, rarely or sometimes (35).

Severely food-insecure: The individuals in the household had to cut back on meal-size or number of meals often, and/or experienced any of the three most severe conditions (running out of food, going to bed hungry, or going a whole day and night without eating) (35).

Low Dietary Diversity; - is three or less type of food groups consumed by the individual respondent over the 24-hour recall period from WDDS food list (36).

Medium dietary diversity score: - Four or five type of food groups consumed by the individual respondent over the 24-hour recall period from WDDS food list .

High dietary diversity score:-Six or more type of food groups consumed by the individual respondent over the 24-hour recall period from WDDS food list (36).

Nutrition knowledge:- Nutrition knowledge of women consists of micronutrient supplements, benefits of folic acid supplements, health risks for low birth weight babies, and birth interval effects on women's nutrition. Five items measuring nutritional knowledge were scored on a dichotomous scale $(0=$ "does not know" response, $1=$ "knows"). For each question, a correct answer was coded as 1 and an incorrect answer as 0 . The total score was a maximum of 5. Mean is used as a cut off value to categorize nutritional status (37).

Dietary change:- Dietary change as a result of current pregnancy was assessed by a single item "Do you change a diet as a result of current pregnancy?"(37).

\section{Data collection tool and procedure}

An interviewer-administered structured questionnaire was used to collect the data for the quantitative part of the study and qualitative data was collected using key informant in-depth 
interview and focus group discussions. The tool included: Socio-demographic factors adapted from the Ethiopian demographic and health survey (EDHS 2016) (38), Maternal related factor, Socio Cultural factors, Economic factors, Hygiene and Sanitation related factors and Food Security related factors.

To determine nutritional status of pregnant mothers, MUAC (mid upper arm circumference) was used. Mid upper arm circumference of the left arm was measured triplicate using a non-stretchable standard MUAC tape to the nearest $0.1 \mathrm{~cm}$ with no clothing on the arm. The mean of triplicate measurement was taken. The value of MUAC bellow $23 \mathrm{~cm}$ was considered as under nourished and MUAC $\geq 23 \mathrm{~cm}$ considered as normal nutritional status $(33,39)$.

Dietary diversity information of individual respondent was collected using 24-hour recall method and women dietary diversity score model questionaries' of nine food groups with food listing method in which list of food items replaced by common foods in local context included in the questioner (40).

House hold food security was measured by house hold food insecurity access scale(HFIAS) which is an adopted approach in estimating the prevalence of food insecurity in the united states(USA) was used to estimate the food insecurity among study participants (35). HFIAS prevalence indicator categorizes households into four level of food security as food secure, mildly, moderately and severely food insecure(24). HFIAS yes or no questions were used to collect information of food security status of the house hold followed by the occurrence of the situation if the response is yes (35). For the occurrence of once or twice it was recorded as rarely, if the occurrence is 3-10 times it was categorized as sometimes and if the occurrence was more than ten times in the past four weeks it was categorized as often $(25,41)$.

Purposively selected subjects were participated in the two focused group discussion for qualitative data. The composition of focus group discussion participants was, pregnant mothers, elders or mother's in-low, and their husbands, health extension workers were engaged in the discussion separately to facilitate the expression of opinions without fear and key informant interview was held with health department head and agricultural expert (agriculture and natural resource head). The discussion was conducted at community meeting places, and the information was collected using open ended questions. Note taking and tape recording were used to document the appropriate information and detect redundant responses. Identified redundant responses were considered to be saturated and removed every evening after triangulation the day work during preliminary analysis. 


\section{Data Quality Assurance}

To assure data quality, training was given for data collectors and supervisors before data collection. The dada collection tool was pre-tested in 5\% of the sample size. The pretest was conducted on individuals having similar characteristics of the study in kebele which was not selected in this study. After pre-test, the instrument was modified accordingly. Supervisors supervised the data collection process and checked the completeness of the questionnaire on a daily base. In addition, principal investigators carefully cleaned the data and entered collected data into a computer software.

\section{Data Processing and Analysis.}

Epi-data version 3.1 statistical software was used for data entry and exported to SPSS version 21 for analysis. Descriptive statistics like mean, standard deviation. frequencies and percentages were computed. Bivariable and multivariable logistic regression was used to determine the degree of association between independent and dependent variables. All variables with p-value less than 0.2 in bivariable analysis were enterred into a multivariable logistic regression. The presence of association between dependent and independent variables was checked with adjusted odds ratio with $95 \%$ confidence intervals. Then the statistical significant was declared at p-value less than 0.05 and adjusted odds ratio interval which excluded one. Assumption of logistic regression such as; meaningful coding, multicollinearity and outliers checking were done before logistic regression model analysis. Multicollinearity was also checked by using Variance inflation factors and Tolerance test. The Hosmer-Lemeshow tests was checked to assess goodness-of-fit model and it was good fit( $\mathrm{p}$-value > 0.05). Wealth index of individual respondent families was also analyzed by using principal component analysis. 


\section{Results}

\section{Socio-demographic characteristics of the respondents}

From the total of 527 pregnant women, 501 participated in this study making the response rate of $95 \%$. The mean age of the respondents was $28.37 \pm 5.246$ years, range between 18-45 years and the majorities of the study subjects were age between 25-34 years. Regarding the marital status, 486(97\%) of the study participants were married and living with their husbands and the rest were unmarried. Among the total, 92(18.36\%) of the pregnant women had husbands who have another wife or polygamous. The average family size of the study subjects was $5.9 \pm 2.74$, with maximum family size of 14 and the minimum of 2 . Majority $335(66.8 \%)$ of the study participants were Protestants religion followers, 95(18.9\%) were Orthodox and the rest were traditional believers. Regarding educational status, 341(68.06\%) were illiterate, $54(10.8 \%)$ were can read and write, $68(13.6 \%)$ were primary, and $38(7.58 \%)$ were secondary and above educational status (Table 1). 
Table 1Sociodemographic characteristics of respondents in Konso district, southern Ethiopia $2019(n=501)$

\begin{tabular}{|c|c|c|c|}
\hline Variable & Category & Frequency & Percent (\%) \\
\hline \multirow[t]{2}{*}{ Marital status } & Married & 486 & 97.01 \\
\hline & Unmarried & 15 & 2.99 \\
\hline \multirow[t]{3}{*}{ Age of women in years } & $18-24$ & 103 & 20.5 \\
\hline & $25-34$ & 323 & 64.5 \\
\hline & $35-45$ & 75 & 15 \\
\hline \multirow[t]{3}{*}{ Family size in number } & $2-5$ & 249 & 49.7 \\
\hline & $6-8$ & 170 & 33.9 \\
\hline & $\geq 9$ & 82 & 16.4 \\
\hline \multirow[t]{3}{*}{ Religion } & Protestant & 335 & 66.9 \\
\hline & Orthodox & 95 & 19 \\
\hline & Cultural believers & 71 & 14.2 \\
\hline \multirow[t]{4}{*}{ Women's educational status } & Illiterate & 341 & 68.1 \\
\hline & Can read and write & 54 & 10.8 \\
\hline & Primary education & 68 & 13.6 \\
\hline & Secondary and Above & 38 & 7.6 \\
\hline \multirow[t]{4}{*}{ Husband educational status } & Illiterate & 273 & 54.5 \\
\hline & Can read and write & 67 & 13.4 \\
\hline & Primary education & 61 & 12.2 \\
\hline & Secondary and Above & 100 & 20 \\
\hline \multirow{4}{*}{$\begin{array}{l}\text { Women's } \\
\text { occupational status }\end{array}$} & Farmer & 397 & 79.2 \\
\hline & Merchant & 42 & 8.4 \\
\hline & Daily worker & 40 & 8 \\
\hline & Government worker & 22 & 4.4 \\
\hline \multirow{4}{*}{$\begin{array}{l}\text { Husband's } \\
\text { occupational status }\end{array}$} & Farmer & 359 & 71.7 \\
\hline & Merchant & 44 & 8.8 \\
\hline & Daily worker & 44 & 8.8 \\
\hline & Government worker & 54 & 10.8 \\
\hline \multirow[t]{2}{*}{ Coo wife for husband } & Yes & 92 & 18.36 \\
\hline & No & 409 & 81.6 \\
\hline \multirow{4}{*}{$\begin{array}{l}\text { Average family income } \\
\text { (Ethiopian birr) }\end{array}$} & $<500$ & 350 & 69.9 \\
\hline & $500-1000$ & 63 & 12.5 \\
\hline & $1001-1500$ & 23 & 4.6 \\
\hline & $>=1501$ & 65 & 12.9 \\
\hline \multirow{3}{*}{$\begin{array}{l}\text { Decision-making power on } \\
\text { house holds asset }\end{array}$} & Husband only & 183 & 36.52 \\
\hline & Wife only & 14 & 2.7 \\
\hline & Husband and wife & 304 & 60.6 \\
\hline \multirow[t]{4}{*}{ Wealth index } & Poor & 392 & 78.2 \\
\hline & Medium & 67 & 13.4 \\
\hline & Rich & 29 & 5.8 \\
\hline & Very rich & 13 & 2.6 \\
\hline
\end{tabular}




\section{Food security status}

Regarding food security status of hold of the study subjects, about 244(44.7\%) were food secured families but the rest are food in secured (Figure 1).

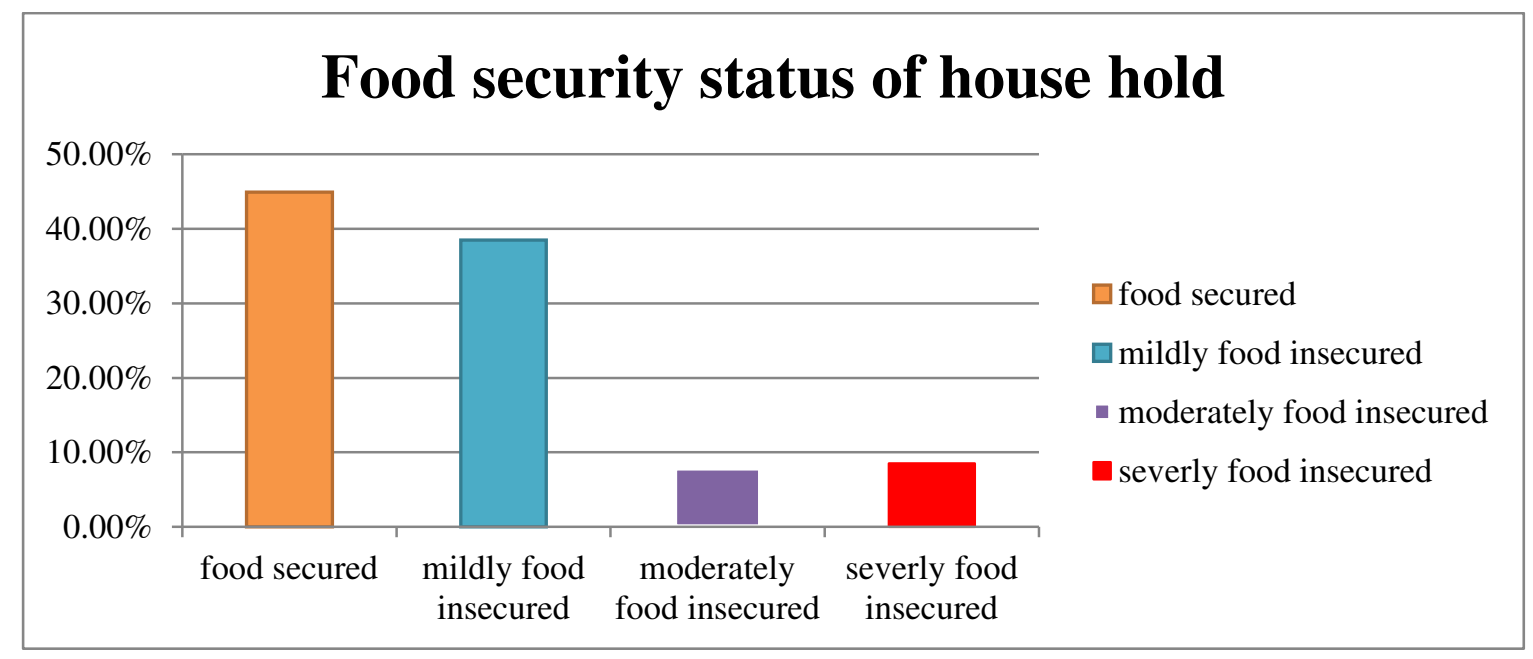

Figure 1 Family Food Security Status of respondents in Konso district, southern Ethiopia, 2019

\section{Maternal related characteristics of respondents}

A total of $359(71.6 \%)$ of mothers received pregnancy related nutrition information from health professionals and health extension workers. Regarding meal frequency, 42(8.38\%) reported eating once a day, 302(60.2\%) of them had reported to eat three times and more a day. Majority $432(86.3 \%)$ of the study participants were multi gravida whereas only 69(13.7\%) were primi-gravida. Regarding gestational age 46(9.18\%) were in first trimester and $304(60.67 \%)$ of pregnant mothers were in third trimesters of gestational age.

From total under nutrition case more than fifty percent of mothers or 134(62.03\%) are reported from mothers in third trimester gestational age. Regarding the birth interval, $141(28.14 \%)$ of mothers reported the interval between children is below 24 months and the rest was above 24 months or more. Bout 336(67.06) of women reported they used modern family planning method before current pregnancy and 105(20.9\%) of women reported that the current pregnancy was not intended. A total of 34(6.78\%) had history of still birth and the rest 467(93.22\%) did not experienced still birth. Those mother had history of abortion where $41(8.18 \%)$ and the rest had never experienced abortion. Regarding ANC attendance majority $339(67.66 \%)$ of women attended antenatal clinic twice or more and $61(12.17 \%)$ of pregnant mothers never attended ANC clinic (Table 2). 
Table 2 Maternal related characteristics of respondents in Konso district, southern Ethiopia $2019(\mathrm{n}=501)$.

\begin{tabular}{|c|c|c|c|c|}
\hline Variable & Category & Under nutr & & Total \\
\hline & & Yes & No & \\
\hline Number of pregnancy & 1 & $29(5.78 \%)$ & $40(7.98 \%)$ & $69(13.77 \%)$ \\
\hline & $2-5$ & $140(27.9 \%)$ & $186(37.12 \%)$ & $326(65.06 \%)$ \\
\hline & $\geq 6$ & $47(9.38)$ & $59((11.77 \%)$ & $106(21.15 \%)$ \\
\hline Historyof family planning & Used & $70(13.97 \%)$ & $95(18.96) \%$ & $165(32.93 \%)$ \\
\hline utilization & Not used & $146(29.14 \%)$ & $190(37.92 \%)$ & $336(67.06)$ \\
\hline Received nutrition & Yes & $154(30.73 \%)$ & $205(40.91 \%)$ & $359(71.65 \%)$ \\
\hline & No & $62(12.37 \%)$ & $80(15.96 \%)$ & $142(28.34 \%)$ \\
\hline Gestational age & $1^{\text {st }}$ trimester & $19(3.7 \%)$ & $40(7.98 \%)$ & $69(13.77)$ \\
\hline & $2^{\text {nd }}$ trimester & $63(12.57 \%)$ & $186(37.12 \%)$ & $326(65.06 \%)$ \\
\hline & $3^{\text {rd }}$ trimester & $134(26.74 \%)$ & $59((11.77 \%)$ & $106(21.15 \%)$ \\
\hline Knowledge of additional & Yes & $170(33.93 \%)$ & $225(44.9 \%)$ & $395(78.84 \%)$ \\
\hline meal during pregnancy & No & $46(9.18 \%)$ & $60(11.97)$ & $106(21.15 \%)$ \\
\hline Antenatal attendance & Yes & $244(48.7 \%)$ & $180(35.9 \%)$ & $424(84.6 \%)$ \\
\hline & No & $41(8.2 \%)$ & $36(7.2 \%)$ & $7715.4 \%)$ \\
\hline History of still birth & Yes & $14(2.8 \%)$ & $20(4 \%)$ & $34(6.8 \%)$ \\
\hline & No & $270(53.9 \%)$ & $197(39.3 \%)$ & $467(93.2 \%)$ \\
\hline Received iron during & Yes & $225(44.9 \%($ & $170(33.9 \%)$ & $395(78.8 \%)$ \\
\hline ANC & No & $60(12 \%)$ & $46(9.2 \%)$ & $106(21 \%)$ \\
\hline
\end{tabular}

\section{Dietary diversity score}

According to dietary diversity assessment result, majority (54\%) of women scored low dietary diversity score or DDS and the rest met the standard dietary diversity score criteria of medium and high (Figure 2).

\section{Diversity score of respondants.}

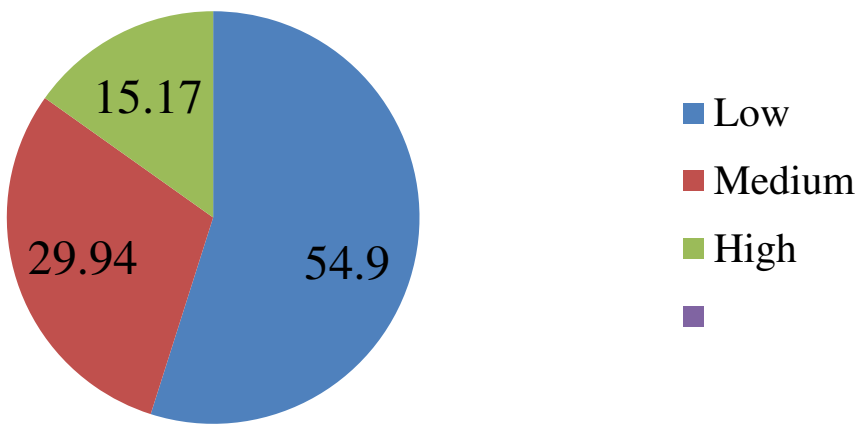

Figure 2 Dietary diversity score of the respondents in Konso district, southern Ethiopia, 2019 


\section{WASH related characteristics of Study participants}

According to water, hygiene and sanitation status of the study subjects, 230(45.9\%) house hold did not have pit latrine and the latrine coverage was only $54.1 \%$. Regarding time taken to fetch water, $134(26.74 \%)$ of mother's were traveling one hour and more to get water and $188(37.52 \%)$ mothers were travelling less than 30 minute to get water. The majority 190 (37.9\%) of respondents was using river as source of water followed by communal water point (Table 3, Figure 3)

Table 3 WASH related Factor of respondents in Konso district, southern Ethiopia, $2019(n=501)$

\begin{tabular}{|l|c|c|c|c|}
\hline \multicolumn{1}{|c|}{ Variable } & Category & \multicolumn{2}{|c|}{ Under nutrition } & Total \\
\hline \multirow{2}{*}{$\begin{array}{l}\text { Place of } \\
\text { defecation }\end{array}$} & Bush & Yes & No & \\
\cline { 2 - 5 } & Community pit & $103(20.55 \%)$ & $101(20.15 \%)$ & $204(40.71 \%)$ \\
\cline { 2 - 5 } & $\begin{array}{c}\text { Use their own } \\
\text { latrine }\end{array}$ & $97(19.36 \%)$ & $174(34.73 \%)$ & $271((54.09 \%)$ \\
\hline \multirow{2}{*}{$\begin{array}{l}\text { Latrine } \\
\text { availability }\end{array}$} & Yes & $99(19.76 \%)$ & $172(34.33 \%)$ & $271(54.09 \%)$ \\
\hline \multirow{2}{*}{$\begin{array}{l}\text { Time to fetch } \\
\text { water }\end{array}$} & No & $117(23.35 \%)$ & $113(22.55 \%)$ & $230(45.90 \%)$ \\
\cline { 2 - 5 } & $30-60$ minutes & $80(15.96 \%)$ & $108(21.55 \%)$ & $188(37.52 \%)$ \\
\cline { 2 - 5 } & $\geq 60$ minutes & $60(11.97 \%)$ & $74(14.77 \%)$ & $139(35.72 \%)$ \\
\hline
\end{tabular}

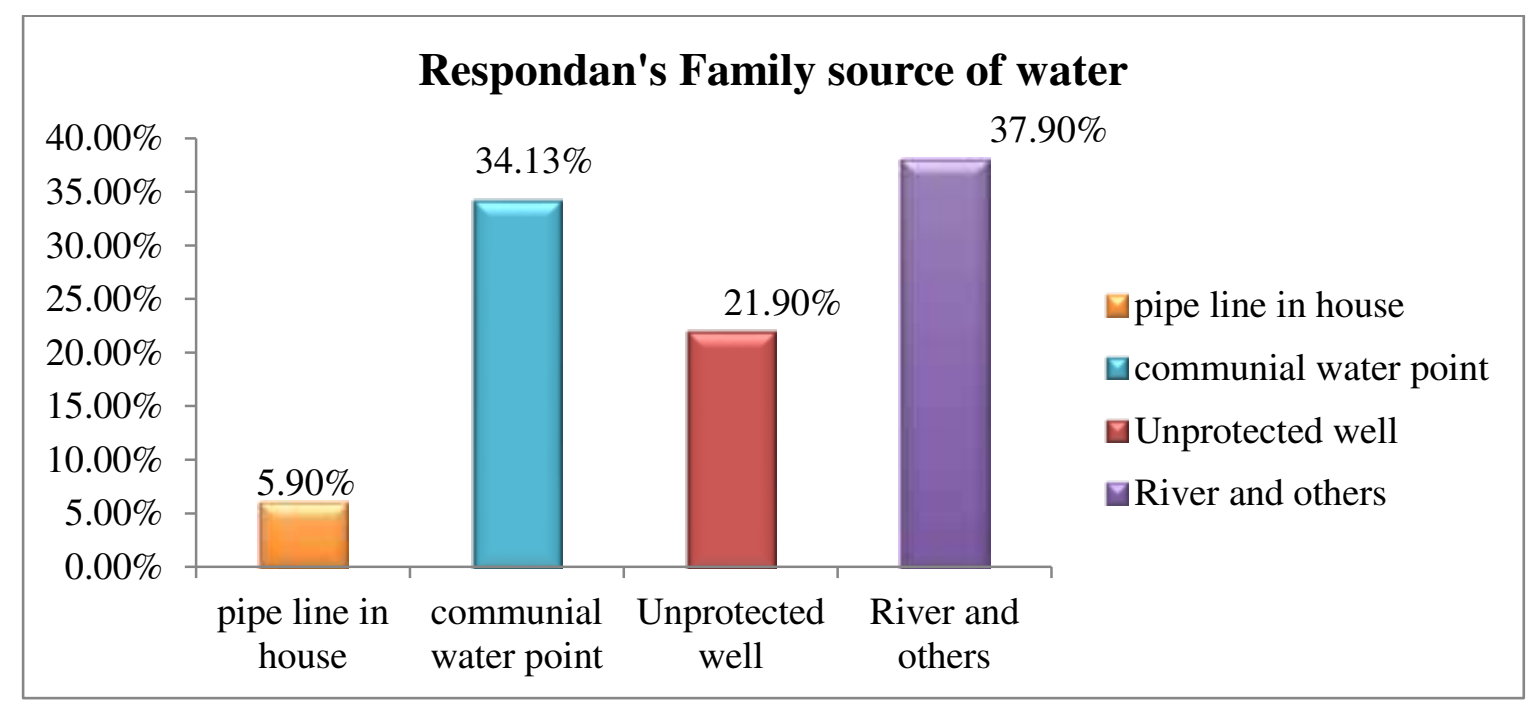

Figure 3 House hold source of water for respondents in Konso district, southern Ethiopia, 2019. 


\section{Prevalence of under nutrition among the study participants.}

The result of this study found, 216(43.11\%) (95\% CI: 38.76\%-47.47) of the pregnant women were undernourished (MUAC $<23 \mathrm{~cm}$ ) and those with MUAC greater than or equal to $23 \mathrm{~cm}$ were $285(56.9 \%$ ) (Figure 4). The mean MUAC was $22.9 \pm 1.43(\mathrm{SD})$, the minimum and maximum MUAC for the study subject were $18 \mathrm{~cm}$ and $26 \mathrm{~cm}$ respectively.

\section{Nutritional status of study participants}

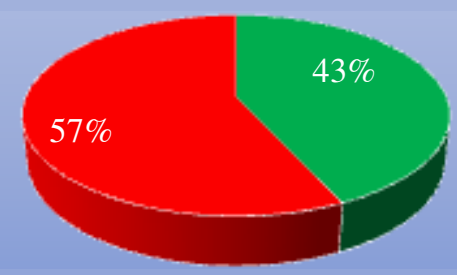

- MUAC less than $23 \mathrm{~cm}$

- MUAC greater than or equal to $23 \mathrm{~cm}$

Figure 4 Nutritional status of respondents in Konso district, southern Ethiopia, 2019.

\section{Factors associated with undernutrition}

Both Bivariable and Multivariable logistic regression analysis were employed. In the Bivariable analysis variables such as: religion, marital status, mothers occupational status, husbands occupational status, average family income, source of water, resource decision making power on house hold asset, family food security status, history of still birth, knowledge of additional meal during pregnancy, latrine availability, dietary diversity score and iron supplementation were significantly associated with undernutrition. Variables with pvalue of $<0.25$ in the Bivariable logistic regression analysis were entered into multivarible logistic regression analysis.

In Multivariable analysis, family food insecurity (AOR = 3.1; 95\%CI: 2.1-4.6), low dietary diversity score $(\mathrm{AOR}=4.9 ; 95 \% \mathrm{CI}$ : 2.6-9.2), medium dietary diversity score (AOR = 2.3; 95\%CI: 1.2-4.7), absence of household latrine (AOR = 1.8; 95\%CI: 1.2-2.6) and having family resource decision making by husband only $(\mathrm{AOR}=1.7$; 95\%CI: 1.1-2.6) were significantly associated with undernutrition (Table 4). 
Table 4 Factors associated with nutritional status of pregnant women in Konso district, Southern Ethiopia, 2019 ( $n=501)$.

\begin{tabular}{|c|c|c|c|c|c|c|}
\hline \multirow[t]{2}{*}{ Variables } & \multirow[t]{2}{*}{ Category } & \multicolumn{2}{|c|}{ Under nutrition } & \multirow{2}{*}{$\begin{array}{l}\text { COR } \\
(95 \% \mathrm{CI})\end{array}$} & \multirow{2}{*}{$\begin{array}{l}\text { AOR } \\
(95 \% \mathrm{CI})\end{array}$} & \multirow[t]{2}{*}{ P-value } \\
\hline & & Yes & No & & & \\
\hline \multirow[t]{2}{*}{ Family food security status } & Secured & 62 & 162 & Ref. & & \\
\hline & Not secured & 154 & 123 & $3.3(2.3-4.7)$ & $3.1(2.1-4.6)$ & $<0.001$ \\
\hline \multirow[t]{3}{*}{ Dietary diversity score } & Low & 148 & 127 & $4.7(2.6-8.4)$ & $4.9(2.6-9.2)$ & $<0.001$ \\
\hline & Medium & 53 & 97 & $2.2(1.2-4.30)$ & $2.3(1.2-4.7)$ & 0.016 \\
\hline & High & 15 & 61 & Ref. & & \\
\hline \multirow[t]{2}{*}{ Latrine availability } & Yes & 99 & 172 & Ref. & & \\
\hline & No & 117 & 113 & $1.8(1.3-2.6)$ & $1.8(1.2-2.6)$ & 0.004 \\
\hline \multirow[t]{3}{*}{ Resource decision making } & Husband only & 89 & 94 & $1.5(1.0-2.4)$ & $1.7(1.1-2.6)$ & 0.011 \\
\hline & Wife only & 7 & 7 & $1.5(0.5-4.44)$ & $1.9(0.6-6.0)$ & 0.295 \\
\hline & Both equally & 120 & 184 & Ref. & & \\
\hline
\end{tabular}

COR:Crude odds ratio, AOR:Adjusted odds ratio, Ref: Reference category, CI:Confidence interval

\section{Focus Group Discussion}

Focus group discussion was held with 12 participants in each two FGD from different community groups such as pregnant mothers, husbands of pregnant mothers, mother in-low and elders. Different barriers related with pregnant mother nutrition in study area were explored during the focus group discussion session. Responses was coded and categorized by content with thematic analysis.

\section{Food restrictions, cultural believe related barriers.}

There were perceived religious or cultural beliefs that can affect the dietary habit of pregnant mothers in the study areas. The practice to avoid some foods with good quality nutrients such as milk, and milk products, egg during pregnancy is common in this setting. One of the study participants said:-

“...Culturally, pregnant women are not allowed to eat egg, butter, milk, other milk products and avocado during pregnancy. The reason why it is not allowed to eat the list of food items above is, we believe when the pregnant mother ate these foods, the baby became very big and our fear is the mother will face difficulty in child birth." A 31years old age pregnant mother 
There is one quote related with using butter in meal during pregnancy and this quote is used when people explaining some think which is not relevant to be discussed or told before it is real time or proud with something which is hope but not at hand. This saying is: "Why you drink butter before the fetus delivered from mother's womb” 31years pregnant woman Another participant said:

“...The practice and attitude of some specific parts of food restriction is currently changing but commonly practiced in illiterate community and traditional believe followers." 35 years FGD Participant

Another participant responded for the time when some foods restricted during pregnancy as:-

“...It is from her early pregnancy or as she knows she is pregnant, mostly since $4^{\text {th }}$ month or second trimester of pregnancy. She also stated, the Cause for physical health deterioration in my opinion is; there is food shortage in our area, but food sharing practice within the family in our community has its own problem. The community gives priority for husbands and elders in food sharing and mothers who engaged in food sharing and small kids left with residual. Sometime the remaining food might not be adequately support mothers in their nutrition and mother will fall in food inadequate." 58 years mother in low

Another participant explained:-

“...Off course our area is drought prone and frequently affected by drought, but besides to that some husbands have not given attention for their wives and family because our community is male dominant and males are the decision makers for all family related issues. on other hands males are mobile (moves from place to place for work related purposes) and can have an opportunity to get variety of foods but mothers and small kids are restricted to be stayed at home so that they lack opportunity to get variety of food." 38 years old pregnant women husband

\section{Food production related barriers}

Konso agriculture and natural resource department staffs interviewed for food security and agricultural production related information of the district and the response was:

"...The area is drought prone frequently affected by drought. The farming is rain dependent and the rain fall pattern is inerratic every year, soil is seriously degraded and the land holding size is also very small which is 0.25-0.5hector per house hold which cannot adequately be the source for family food" Agriculture and natural resource office head “...Food shortage is common in our district, Currently the district is categorized as priority one district in early warning, four thousand six hundred twenty-seven people included in 
safety net program,31570 people are exposed to emergence, almost one third of the population are in food insecurity status. "' Agriculture and natural resource department head

\section{Food diversification practice related barriers.}

Food diversification practice among study participants has been observed and the practice was poor besides nutrition information provided by community health workers.

One of the study participants said:-

“...The health extension workers in the community are giving nutrition education in food diversification. However, nutrition counseling up take and implementation by our mothers is compromised, almost all households have chicken, producing egg and fruits grown in the garden but, most of them used to sell for house hold income rather than used for consumption. This indicates poor nutritional status of pregnant women is not only associated with lack of food, but also diversification of food is not commonly practiced" community health worker

"...The feeding practice in our community is little bit different, the food content is not diversified, taking snacks and additional meal during pregnancy is not common and nutrition education activities at community level is not strong besides to very low socio economic status of the community" community health workers

“...Family meal preparation trend is also totally changed in my observation, the meal for family is only prepared once at night and the whole day people are depending on locally prepared drink called Chaka including pregnant mothers itself." community health workers

Again he said, "Our district is supported by therapeutic supplementary feeding program for pregnant women and lactating mothers and children under five years of age, but community level health services such as screening mothers and children for their nutritional status is not strong." community health workers 


\section{Discussion}

The purpose of this study was to assess the prevalence and associated factors of undernutrition among pregnant women in Konso district, southern Ethiopia. This study found that four in every ten pregnant women were undernutrished. Food security, dietary diversity, latrine availability, family resource decision making were significant determinants of undernutrition. Food restriction practices, weak nutrition education and malnutrition screening program, practice of depending on local alcoholic drink called "Cheka", draught, rain dependent farming practices and low socio-economic status were identified barriers from qualitative data.

The current study found that the prevalence of undernutrition in pregnant women was 43.11\% (95\% CI: 38.76\%-47.47. The result of this study is higher than those studies conducted in other areas of Ethiopia, Central Refit Valley of Ethiopia, Wondogenet District,southern ethiopia, Silte zone souther Ethiopia, Dessie town, northeastern Ethiopia. Eastern Ethiopia, humanitarian setting in Ethiopia, Gondar hospital and town, 31.8\%, 9.2\%, $21.8 \%, 19.8 \%, 19.5 \%, 24 \%, 16.2 \%$ and $14.4 \%(13-20)$. The discrepancy might be due to difference in sociodemographic characterstics, geographical variation, cultural belives such as food taboos, poor nutritional intervention program in Konso district. Another possible reason may be due to a difference in season of studies conducted.

The finding of this study is also higher than the report from Madagaskar, systematic review in Africa, South Sudan,Keniya, China,India, Sirlamka, Nigeria 9\%, 18.9\%, 19.3\%, $23.5 \%, 11.8 \%, 5 \%, 15 \%, 10 \%(10,12,23,42-46)$. This variation might be due to differences in MUAC cut of value, and the socio-culture distinctions between Ethiopia and the other counties

The family food security status was significantly associated with pregnant women undernutrition. The odds of undernutrition among pregnant women who had family food insecurity status were three times higher than with those women who had secured family food $(\mathrm{AOR}=3.1 ; 95 \% \mathrm{CI}: 2.1-4.6)$. This finding is similar to previous study conducted in Gambella and Arbaminch Zuria Woreda Ethiopia $(36,47)$. This could be possibly due to that family food shortage usually results in lack of daily nutritional requirement and poor dietary intake leading to undernutrition of women. This finding is also consistent with the information given from one key informant: - "...The area is drought prone and frequently affected by drought. The farming is rain dependent and the rain fall pattern is erratic every year, soil is seriously degraded and the land holding size is also very small which is 0.25 - 
0.5hector per house hold which cannot adequately be the source for family food" Agriculture and natural resource office head

Dietary diversity score was one of the factor significantly associated with under nutrition among pregnant mothers. The odds of undernutrition among pregnant women with low dietary diversity score were about five times higher when compared with those women with high dietary diversity score $(\mathrm{AOR}=4.9 ; 95 \% \mathrm{CI}$ : 2.6-9.2) and the odds of undernutrition among pregnant women with medium dietary diversity score were two times higher when compared with those women with high dietary diversity score $(\mathrm{AOR}=2.3 ; 95 \% \mathrm{CI}$ : 1.2-4.7). This result is in line with the study conducted in Gambella Ethiopia (36). This might be due to the fact that mothers who have practice of food diversity will get different nutrient from different diet and this might causes them to be well nourished than those with less than average dietary diversity score.

The result is also consistent with data from focus group discussion; the traditional believe that restrict to consume some parts of foods with high proteins and energy providing foods such as butter, milk, milk products, egg and avocado during pregnancy. As one of the participant said:-“...Culturally, pregnant women are not allowed to eat egg, butter milk, other milk products and Avocado during pregnancy. The reason why it is not allowed to eat the list of food items above is, we believe when the pregnant mother ate these foods, the baby became very big and our fear is the mother will face difficulty in child birth.' 31years old FGD Participant. There for, this can also affect dietary diversity of pregnant women and lead to poor nutritional status of the pregnant mothers.

Latrine availability in the household level was significantly associated with undernutrition of pregnant women. The odds of undernutrition among pregnant women without latrine in their house were 1.8 times higher when compared the counterparts (AOR $=1.8$; 95\%CI: 1.2 2.6). (48). The possible explanation may be those pregnant women who have no latrine and using open field defecation have higher exposure for recurrent diarrheal disease infection this may lead to poor nutritional status of the pregnant mothers since infection and malnutrition have direct relationship and it is an immediate cause for under nutrition.

The decision making power on house hold assets had also significant association with pregnant women undernutrition. The odds of undernutrition among pregnant women who had family resource decision making by husband only were 1.7 times higher than those who made decision both husband and wife (AOR $=1.7$; 95\%CI: 1.1-2.6). This finding is in line with findings from qualitative study conducted in three food insecure districts of Tigray Region, Northern Ethiopia (49). This might be due the reason that one part wife only or husband only 
decision in family resources may affect their communication and if one ignores the idea of others, this may cause the wives not to be supported by their husbands and this may also negatively affect their nutritional habit. In addition to that the finding was consistent with qualitative data from focus group discussion:- “...Some husbands have not given attention for their wives and family because our community is male dominant and males is the decision makers for all family related issues. On other hands males are mobile (moves from place to place for work related purposes) and can have an opportunity to get variety of foods but mothers and small kids are restricted to be stayed at home so that they lack un opportunity to get variety of food. " FGD participant

\section{Limitations of the study}

This study recognized the following limitations: Some self-reported variables like household food security status and decision making power on house hold asset may be affected by social desirability bias and it was reduced through detail clarification of the objective before entering into individual interview. In dietary diversity assessment since 24-hour recall data collection (food listing method) used, through interview process recall bias is expected (reduced by probing).

\section{Conclusions}

The prevalence of under nutrition among pregnant women was found to be higher than previously reported findings in Ethiopia and other countries. Food security, dietary diversity, latrine availability, family resource decision making, food restriction, weak nutrition education and malnutrition screening program, practice of depending on local alcoholic drink called "Cheka", draught, poor hygiene and sanitation coverage ,traditional way of farming and low socio-economic status were identified factors. Hence, interventions targeting maternal nutrition education, personal hygiene and sanitation, encouraging irrigation through working with agricultural sector to change traditional way of farming practices and the economic status of community are recommended.

\footnotetext{
Abbreviations

ANC: Antenatal care; DD: Dietary diversity; DDS: Dietary diversity score; EDHS: Ethiopian Demographic and Health Survey; FAO: Food and Agriculture Organization; FS: Food security; IUGR: Intrauterine Growth Retardation; IFAD: International fund for agriculture and development; MUAC-Mid-Upper Arm Circumference: SPSS----Statistical Package for Social Science; WFP: World Food Program; WHO: World Health Organization
} 


\section{Ethical approval and consent to participate}

Ethical clearance was obtained from ethical review committee of Arba Minch University, college of Medicine and Health sciences with reference number IRB/12032569/111. From Konso district healgh office, letters of permission were obtained for each selected kebele. Written informed consent was obtained from each participant at the time of data collection and for those illiterate participates the informed consent was obtained from the legal guardian. Those individuals not willing to participate were given the right to do so. Throughout all research process, confidentiality of response was also ensured. All methods were carried out in accordance with relevant guidelines and regulations in method section

\section{Consent for publication}

Not applicable.

\section{Availability of data and materials}

All relevant data are available from the corresponding author upon reasonable request

\section{Competing interests}

The authors declare that they have no competing interests

\section{Funding}

Funding was obtained from Arba Minch University, college of Medicine and Health sciences

\section{Authors' Contribution}

DG: Designed and participated in data collection, conducted the data analysis and interpretation, developed the first draft and revised subsequent drafts. GA: advised on the conception of study area, data analysis and interpretation reviewed and commented on successive drafts. DA: advised on the conception of study area, data analysis and interpretation reviewed and commented on successive drafts. MA: Advised on the data analysis and interpretation and commented on successive drafts. All authors reviewed and approved the final manuscript

\section{Acknowledgements}

The authors acknowledge the Arba Minch University, college of Medicine and Health sciences for funding and respondents in Konso district for providing data. We also would like to acknowledge data collectors and supervisors for accomplishing of their tasks.

\section{References}


1. Heyam F. Dalky P, RN1, Abeer Qandil, PhD, RN1 \& Amani A. Alqwasmi, MSN, RN2 1 Factors Associated With Undernutrition Among Pregnant and Lactating Syrian Refugee Women in Jordan 2018;10,No4;2018.

2. Prajakta Ganesh Joshi1* GAJ, Sapna Jain1, Vijaya Dubey3 , , . Nutritional status of pregnant women reporting at Rural Health Training Centre International jornal of reproduction ,conteraception ,obystetric and Gynacology. 29July 2017;6(9):3846.

3. UNICIEF. The lancet series on maternal and child under nutiriton. 2018.

4. Skinner $A-L$, , . Pregnancy outcome in South Asian women: Factors

affecting diet and nutrition 2012.

5. Gemeda Daba FB, Habtamu Fekadu*, Wondu Garoma Assessment of Knowledge of Pregnant Mothers on Maternal Nutrition and Associated Factors in Guto Gida Woreda, East Wollega Zone, Ethiopia Nutrition and food science 2013

6. Ozanne1 MSM-GaSE. Maternal nutrition during pregnancy and health of the offspring. 2006;34, part5

7. Aamer Imdad ZAB. Maternal Nutrition and Birth Outcomes: Effect of Balanced ProteinEnergy Supplementationppe_1308 178.. 2012.

8. Bagriansky J CN, Pak K, Whitney S, Laillou A. The economic consequences of malnutrition in Cambodia, more than 400 million US dollar lost annually. . Asia Pac J Clin Nutr. 2014;23(4):524-31.

9. Lewit EM BL, Corman H, Shiono PH. The direct cost of low birth weight. Futur Child. 1995;5(1):35-56.

10. A.M.N.T. Adikari* RS, D.G.N.G. Wijesinghe2 and C. Liyanage3 , , . Assessment of Nutritional Status of Pregnant Women in a Rural Area in Sri Lanka Tropical agriculture research. 2016;27(2):203-211(2016).

11. Fang-Li Liu1 YMZ, Gerard Vinyes Parés2, Kathleen C Reidy3, Wen-Zhi Zhao1, Ai Zhao1, Cheng Chen1, Celia Y Ning2, Ying-Dong Zheng4, Pei-Yu Wang1 Nutrient Intakes of Pregnant Women and their Associated Factors in Eight Cities of China: A Cross-sectional Study. Chines Medical jornal. July 5/2015;128(13).

12. EA U. Nutritional Practices and Taboos Among Pregnant Women Attending Antenatal Care at General Hospital in Kano, Northwest Nigeria. 2016.

13. Dibaba AFMaB. Epidemiology of Malnutrition among Pregnant Women and Associated Factors in Central Refit Valley of Ethiopia, 20162016.

14. Kuch Desalegn Sp, Moges Debebe. Dietary practice and associated factors among pregnant Women in Wondogenet District,southern ethiopia: a cross-sectional study. Jornal of pharmacetical and scientific in. 06/10/15.

15. Mohammed Muze MY, Shemsu Kedir and Abdilmejid Mustefa. Prevalence and associated factors of undernutrition among pregnant women visiting ANC clinics in Silte zone, Southern Ethiopia. BMC Pregnancy and Childbirth 2020;20(707).

16. TZ D. . Factors associated with dietary practice and nutritional status of pregnant women in Dessie town, northeastern Ethiopia: a communitybased cross-sectional study. BMC Pregnancy Childbirth. 2019;19(1).

17. Kedir H BY, Worku A. Magnitude and determinants of malnutrition among pregnant women in eastern Ethiopia: evidence from rural,community-based setting. Matern Child Nutr. 2016;13(1):51-63.

18. Dadi AF DH. Undernutrition and its associated factors among pregnant mothers in Gondar town, Northwest Ethiopia. plos One. 2019;14(4).

19. Kumera G GD, Alebel A, Feyera F, Eshetie S. Undernutrition and its association with sociodemographic, anemia and intestinal parasitic infection among pregnant women attending antenatal care at the University of Gondar Hospital, Northwest Ethiopia. . Matern Heal Neonatol Perinatol 2018;4(18). 
20. Gebre B BS, Taddese Z, Legesse T, Letebo M. Determinants of malnutrition among pregnant and lactating women under humanitarian setting in Ethiopia. BMC Nutr. 2018;4(1).

21. Belete Y1 ', Nega B2 Firehiwot M3. Under Nutrition and associated factors among adolesent pregnant women in shashemene, west Arsi Zone,Ethiopia: a communiy based study. ornl of nutiriton and food science. January 14,2016.

22. Hutter I. Reduction of food intake during pregnancy in rural South India 1996;1 no 3 pp 399405.

23. Soghra Abasizadeh1 ZH, Fatemeh Deres3 Prevalence of malnutrition during pregnancy and associated factors in women of Ardal County in 2012-2013 2016.

24. Palanivel Chinnakali1 RPU, Deepa Shokeen3, Kavita Singh4, Manpreet Kaur5, Arvind K. Singh6, Anil Goswami6, Kapil Yadav6, Chandrakant S. Pandav6. Prevalance of house hold food insecurity and its determinants in an Urban resetIment colony in North India. 2014.

25. liyas ADZTSSAWAIUDA. Factors affecting house hold food security in rural northern hinterland of Pakistan. Jornal of the Saudi Society of Agricultural science. October 2016.

26. KAHANYA KW. DIETARY DIVERSITY, NUTRIENT INTAKE AND NUTRITIONAL STATUS AMONG PREGNANT WOMEN IN LAIKIPIA COUNTY, KENYA 2013.

27. Sun Eun Lee1 SAT, Mario Merialdi2 and Laura E Caulfield1, * Dietary intakes of women during pregnancy in low- and middle-income countries. 2012.

28. Haidar J. Prevalence of Anaemia, Deficiencies of Iron and Folic Acid and Their Determinants in Ethiopian Women. August 28,2010.

29. Sherin Daniel1* GPSG, Emershia Sharmine3 ,. Effect of nutrition education among pregnant women with low body mass index: a community based intervention 2016.

30. Shiva Raj Acharya1 JB, Diwash Prasad Timilsina3 Factors associated with nutritional status of women of reproductive age group in rural, Nepal. 2017.

31. Hansson F. Maternal factors, early pregnancy anthropometry and gestational weight gain. . 2016.

32. report Kdho. Pregnant women undernutrition. 2018.

33. Siwzerland M. Anthropometric indicators to identify a pregnant woman as acutely malnourished and predict adverse birth outcomes. 1 January 1995 to 12 September 2012

34. Gina Kennedy TBa, Dop M. Guidelines for measuring household and individual dietary diversity. 2013.

35. Coates J, Swindale A, Bilinsky P. Household Food Insecurity Access Scale (HFIAS) for

Measurement of Food Access: Indicator Guide VERSION 3. 2007.

36. Mamo Nigatu TTG, and Desta Hiko Household Food Insecurity, Low Dietary Diversity,

and Early Marriage Were Predictors for Undernutrition among

Pregnant Women Residing in Gambella, Ethiopia. August 2018.

37. Oh HK KS, Cho SH, Ju Y, Faye D. Factors influencing nutritional practices among mothers in Dakar, Senegal. PLoS One. 2019;14(2).

38. Central Statistical Agency [Ethiopia] and ICF International. Ethiopia Demographic and Health Survey. Addis Ababa, Ethiopia, and Calverton. Maryland, USA: Central Statistical Agency and ORC Macro. 2016.

39. Alice M. Tang MC, Kimberly Dong, Norma Terrin, Andrew Edmonds, Nega, Assefa TC, Roshan Ramlal, Parul Christian, Keith West, Naveed Janjua,, Christine Wanke MD, Zeina Maalouf-Manasseh. Determining a Global Mid-Upper Arm Circumference Cutoff to Assess

Malnutrition in Pregnant Women. June 2016. 
40. the Food and Agriculture Organization of the United Nations and USAID's Food and Nutrition Technical Assistance III Project (FANTA) mbF. Minimum Dietary Diversity for Women -A Guide to Measurement. 2016.

41. Amy Webb Girard a, b Julie L. Self,a,b Corey McAuliffe,a Olafunke Oludea The Effects of Household Food Production Strategies on the Health and Nutrition Outcomes of Women and Young Children: A Systematic Reviewppe. 2012.

42. Ravaoarisoa L RL, Rakotonirina J, Rakotomanga J, Donnen P, Dramaix M. Socioeconomic determinants of malnutrition among mothers in the Amoroni mania region of Madagascar: a crosssectional study. BMC Nutr. 2018;4(1).

43. Desyibelew HD DA. Burden and determinants of malnutrition among pregnant women in Africa: A systematic review and meta-analysis. . PLoS One. 2019;14(9).

44. Alemayehu A GL, Yemane T, Asres Y. Prevalence, severity, and determinant factors of Anemia among pregnant women in south Sudanese refugees. Pugnido, Western Ethiopia Anemia. 2016;2016:9817358.

45. Willy KJK PC. Dietary diversity, nutrient intake and nutritional status among pregnant women in Laikipia County, Kenya. Int J Health Sci Res. 2016;6(4):378-85.

46. Gao H SC, Scherbaum V, Biesalski HK, Wang Q, Hormann E, et al. Dietary intake and food habits of pregnant women residing in urban and rural areas of Deyang City, Sichuan Province. China Nutr. 2013;5(8):2933-54.

47. Hadiya Hassen Tikuye1 SG, Addisalem Mesfin1, Susan, Whiting. Prevalence and Factors Associated with Undernutrition among

Exclusively Breastfeeding Women in Arba Minch Zuria District,

Southern Ethiopia: A Cross-sectional Community-Based Study. January1/2019.

48. Mtumwa AH1 BG, Msc.Biostatistics; Paul E1 BA.Statistics, MA.Statistics; Vuai SAH2

Msc.Marine and Environmental Sciences PiMaES. Determinants of undernutrition among women

of reproductive age in Tanzania mainland. 28 Jul 2016.

49. Afework Mulugeta Bezabih $1 \mathrm{MHW}$, * , Znabu Hadush Kahsay 1, Zewditu Getahun 2 and Alessandra N. Bazzano 3. Demand and Supply Side Barriers that Limit the Uptake of Nutrition Services among Pregnant Women from Rural Ethiopia: An Exploratory Qualitative Study. 2018. 
Figures

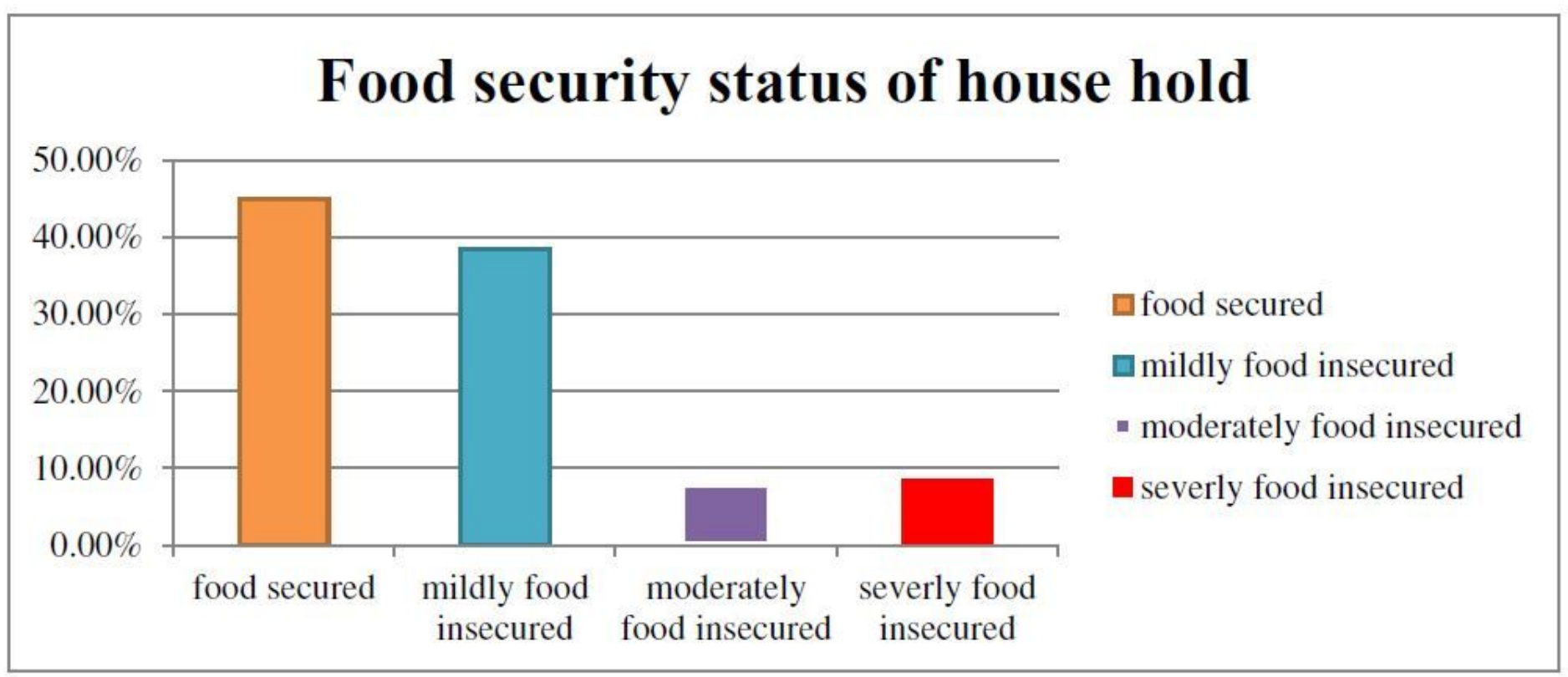

Figure 1

Family Food Security Status of respondents in Konso district, southern Ethiopia, 2019

\section{Diversity score of respondants.}

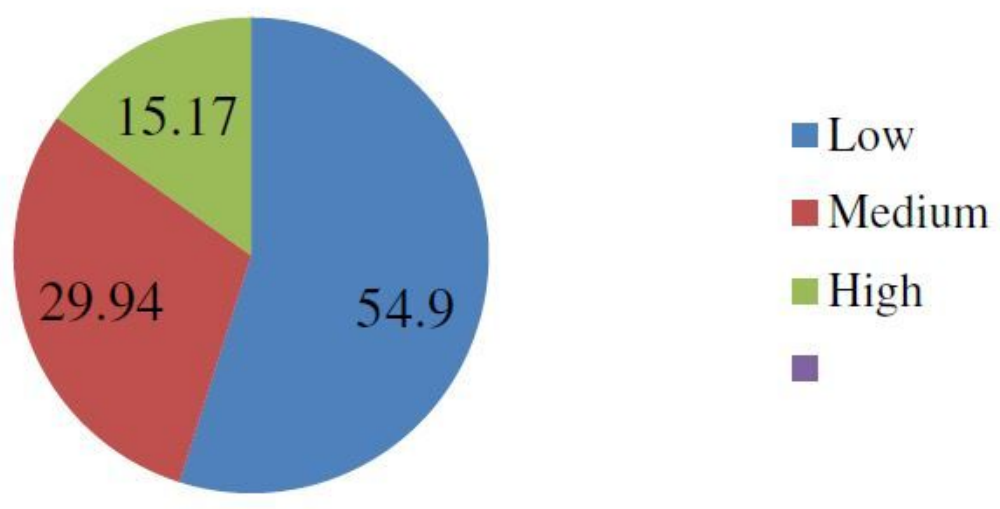

Figure 2

Dietary diversity score of the respondents in Konso district, southern Ethiopia, 2019 


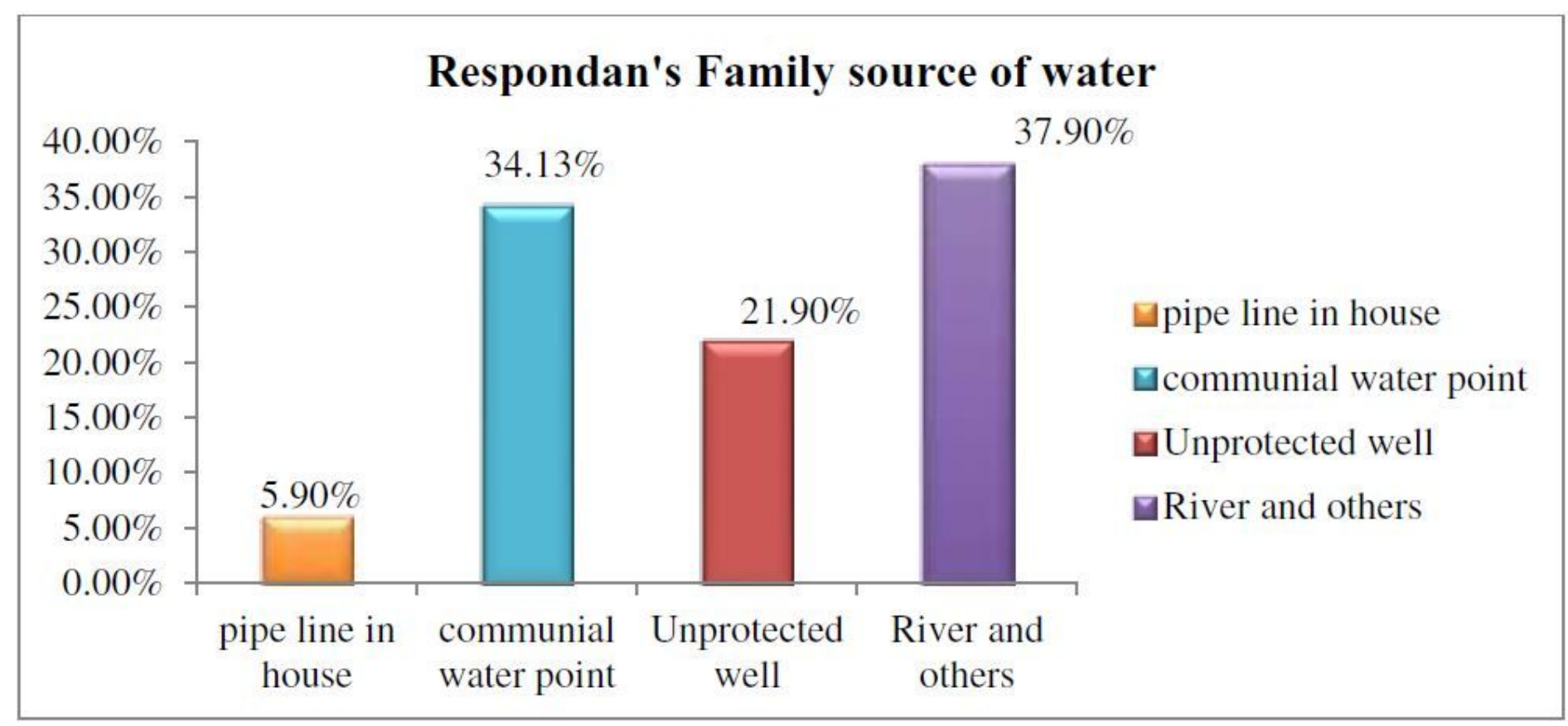

\section{Figure 3}

House hold source of water for respondents in Konso district, southern Ethiopia, 2019.

\section{Nutritional status of study participants}

\section{Figure 4}

Nutritional status of respondents in Konso district, southern Ethiopia, 2019. 